\title{
Production of Thermal Axions across the ElectroWeak Phase Transition
}

\author{
Fernando Arias Aragón ${ }^{a}$ \\ a Instituto de Física Teórica UAM/CSIC and Departamento de Física Teórica, \\ Universidad Autónoma de Madrid, Calle Nicolás Cabrera 13-15, Cantoblanco E-28049 Madrid, Spain \\ E-mail: fernando.arias@uam.es
}

Light axions can potentially leave a cosmic background just like the neutrinos. We complete the study of thermal axion production above the QCD phase transition by providing a smooth and continuous treatment across the electroweak phase transition. Focusing on flavour conserving axion couplings, we quantify axion production due to scattering of thermal bath particles and we include for the first time the contributions from the longitudinal components of the $\mathrm{W}$ and $\mathrm{Z}$ bosons. We perform a model independent analysis in terms of axion effective coupling to heavy quarks, and we also study the predictions for particular QCD axion scenarios, the so-called Minimal Flavour Violating Axion, as well as the invisible axion KSVZ and DFSZ models. Finally, we explore the possibility of testing with cosmological observations the solar axion interpretation of the XENON1T excess. We study minimal scenarios with a detectable signal in future CMB surveys: axions coupled democratically to all fermions, axion-electron coupling generated radiatively and the DFSZ framework for the QCD axion.

40th International Conference on High Energy physics - ICHEP2020

July 28 - August 6, 2020

Prague, Czech Republic (virtual meeting) 


\section{Motivation}

When building frameworks Beyond the Standard Model (BSM) to address some of the SM open problems it is common that new light or massless degrees of freedom arise. Among the many different candidates for new light states, axions are one of the best motivated extensions of the SM, which arose originally as a solution to the Strong CP Problem [1-3]. The Strong CP Problem is the name that receives the astonishing cancellation needed for the predicted value of the $\bar{\theta}$ parameter, which encodes $\mathrm{CP}$ violation in the strong sector as well as the one coming from the quark mass matrices, that in the SM is related to the neutron electric dipole moment (nEDM) $d_{n}$ according to the following expression [4]:

$$
d_{n} \sim \bar{\theta} \times O\left(10^{-16}\right) e \cdot \mathrm{cm} .
$$

Current measurements of the nEDM [5, 6] bound the theta parameter to be at the order $\bar{\theta} \leq O\left(10^{-10}\right)$, which is surprisingly small for a dimensionless parameter. The axion dynamically solves this and, additionally, was found to be a possible dark matter (DM) candidate [7], appearing also naturally within string theories [8]. On top of this, axions are one possible explanation to the observed excess by the XENON1T collaboration [9], making it a very phenomenologically interesting object to study. Axions are currently being searched for following many different strategies: from helioscopes like CAST [10] or IAXO [11, 12], haloscopes like ADMX [13, 14], light shining through wall experiments or indirectly through stellar cooling. In addition to this, there exists the possibility of detecting effects due to the existence of axions through a cosmological probe: the effective number of neutrinos. If axions are produced hot and behave like dark radiation they can leave an imprint on the relativistic degrees of freedom in the universe, that can be parametrized as a modification to the effective number of neutrinos and is related to the axion abundance, $Y_{a}$, as follows:

$$
\Delta N_{\text {eff }} \simeq 74.85 Y_{a}^{4 / 3} .
$$

This cosmological observable is expected to be measured with increased precision in the future by CMB-S4 [15], LiteBIRD [16] and the Simons Observatory [17], allowing to study the axion couplings and decay constant through its effect on $\Delta N_{e f f}$. This dependence is the main object of study in this text, analyzing different production processes and models.

\section{Setup}

Axion interactions with the SM degrees of freedom can be parametrized in the following way:

$$
\mathcal{L}_{\text {axion int }} \supset \frac{1}{f_{a}}\left(c_{X} \frac{\alpha_{X}}{8 \pi} a \tilde{X}_{\mu \nu}^{a} X^{\mu \nu a}+c_{\psi} \partial_{\mu} a \bar{\psi} \gamma^{\mu} \psi\right),
$$

where $a$ is the axion field with its so-called decay constant being $f_{a}$ and $c_{\psi}$ and $c_{X}$ its coupling constants to fermions and gauge bosons respectively, $X$ stands for any SM gauge boson, with $\tilde{X}_{\mu \nu}^{a}=\frac{1}{2} \varepsilon_{\mu \nu \rho \sigma} X^{\rho \sigma a}$ its dual and $\alpha_{X}$ its associated gauge coupling constant, and $\psi$ can be any of the SM fermion fields. The processes where the axions are produced are temperature dependent and will lead to efficient production when they exceed the Hubble rate, which depends on $T$ as $H \propto T^{2}$ during radiation domination. They can be divided in three categories, depending on the SM particles involved: $X+X \rightarrow X+a$, with only gauge bosons. These processes exist both above 
and below the Electroweak Phase Transition (EWPT) and their production rate can be parametrized as:

$$
\Gamma \simeq \alpha_{X}^{3} \frac{c_{X}^{2}}{f_{a}^{2}} T^{3} .
$$

The dominant contribution is that due to gluons and, as $\Gamma / H$ grows with $T$, they are specially relevant at high temperatures where, if thermalization is achieved, $\Delta N_{e f} \simeq 0.027$ can be reached. Following the process categorization, we find now the processes $\psi+X \rightarrow \psi+a, \psi+\bar{\psi} \rightarrow X+a$, involving fermions and gauge bosons. These processes lead to the following production rate for temperatures above the fermion mass, $T>m_{\psi}$ :

$$
\Gamma \simeq \alpha_{X} m_{\psi}^{2} \frac{c_{\psi}^{2}}{f_{a}^{2}} T
$$

For this reason, theses processes only exist below the EWPT and, additionally, for temperatures smaller than $m_{\psi}$ they are Boltzmann suppressed, with $\Gamma / H$ peaking at around $T \sim m_{\psi}$, being dominated by gluons and heavy fermions.

The last class of processes is $\psi+H \rightarrow \psi+a, \psi+\bar{\psi} \rightarrow H+a$, for processes that involve the Higgs doublet. In this case, both above and below EWPT these processes will be present: below they will involve the physical Higgs and the longitudinal components of the $\mathrm{W}$ and $\mathrm{Z}$ bosons, whereas above any component of the Higgs doublet can appear in the process. For masses below the fermion mass Boltzmann suppression will take place, whereas for higher temperatures the production rate is

$$
\Gamma \simeq y_{\psi}^{2} \frac{c_{\psi}^{2}}{f_{a}^{2}} T^{3},
$$

with $y_{\psi}$ being the fermion Yukawa. These processes are also dominated by the heavy fermion contributions and can thermalize at high temperature as well.

\section{Model Independent Results}

Using the previously listed production processes, we will show in Fig. 1 the predicted effect on $\Delta N_{\text {eff }}$. In order to do so, we follow a model independent approach, sticking to an operator-byoperator analysis considering only one axion coupling at a time. Solving the Boltzmann equation downt to $1 \mathrm{GeV}$, so as to stay in the perturbative regime, a prediction for the effective number of neutrinos as a function of the axion scale is obtained. This result is in fact sensitive to the conditions in the Early Universe, as an initial abundance for the axion must be assumed; in our case we assumed thermal initial abundance or zero, the latter leading to a dependence also on the reheating temperature.

\section{UV Complete Models}

In this section we will explore specific models that predict precise axion couplings, leading to a single prediction for $\Delta N_{\mathrm{eff}}\left(f_{a}\right)$ shown in Fig. 2. In particular, we will consider the two classical invisible axion scenarios, namely the DFSZ [18, 19] and $\operatorname{KSVZ}[20,21]$ models. In the former, the SM is extended by the addition of a second Higgs field and a new singlet scalar. In this model, 


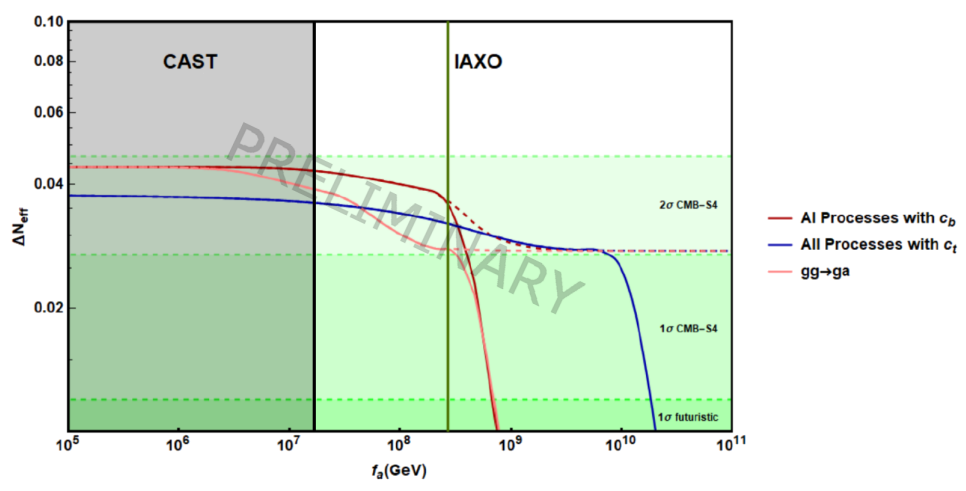

Figure 1: Prediction for $\Delta N_{\mathrm{eff}}$ as a function of $f_{a}$. All different processes involving one same axion coupling are considered within each line. Solid lines represent the prediction when assuming zero initial abundance and $T_{R H}=$ $10^{4} \mathrm{GeV}$, whereas dashed lines were obtained considering thermal initial abundance. The grey shaded region delimited by a black line corresponds to the parameter space ruled out by CAST whereas the vertical green line shows the highest value for $f_{a}$ IAXO is expected to explore, assuming in both cases the axion-photon coupling to be 1 .

the up-type and down-type quarks couple differently to axions, while satisfying that their couplings add up to $1 / 3$ and, depending on to which Higgs doublet charged leptons couple, the anomaly ratio $E / N$ can display two possible values. In the latter, however, the SM is extended only with a new complex scalar singlet and heavy quarks, being the new fields the only with PQ charge. For this reason, the only relevant process is the purely gluonic one and, depending on the representation of these heavy quarks many values of $E / N$ can be obtained.

In addition to these two well known models, we will also consider the prediction given by a specific flavourful axion model: the Minimal Flavour Violating Axion [22], where the MFV assumption was followed and the PQ symmetry was identified within the MFV flavour symmetry group. This predicted a zero coupling of up-type quarks to the axion, as well as $E / N=8 / 3$.

\section{Complementarity with XENON1T}

Finally, in this section we will comment on how axions compatible with the excess measured by the XENON1T collaboration could leave an imprint on the Early Universe through $\Delta N_{\text {eff }}$ [23], showing the results in Fig. 3. When the required axion-electron coupling is taken into account, it is possible to translate it into an expected $\Delta N_{\text {eff }}$ produced by these light relics. In particular, we will be considering three different scenarios. First, we consider a non-anomalous ALP with democratic flavour conserving couplings to all fermions $c_{\psi}=1$. In this case, the signal in $\Delta N_{\text {eff }}$ is dominated by axion-heavy quark scattering and, for the window in the axion scale relevant for XENON1T we find $\Delta N_{\text {eff }}$ to be slightly above 0.04 . The second scenario is one where the axion-electron coupling is radiatively induced from an axion-fermion coupling $(\psi=\tau, c, b$ or $t)$ at the UV scale. For each case, we assume a single $c_{\psi}(f)$ to be nonzero at the UV scale and solve the Boltzmann equation including all the radiatively induced couplings at low energy. When only the top coupling exists in the UV, the induced couplings are relevant and therefore accounted for when solving the Boltzmann equation, yielding $\Delta N_{\text {eff }} \sim 0.04$. For the bottom and charm case, the axion remains in equilibrium down to $1 \mathrm{GeV}$, where we stop the equation solving. Due to this, the value of $\Delta N_{\mathrm{eff}} \sim 0.044$ should be interpreted as a lower bound on the signal. Finally, the tau case can be solved down to much 

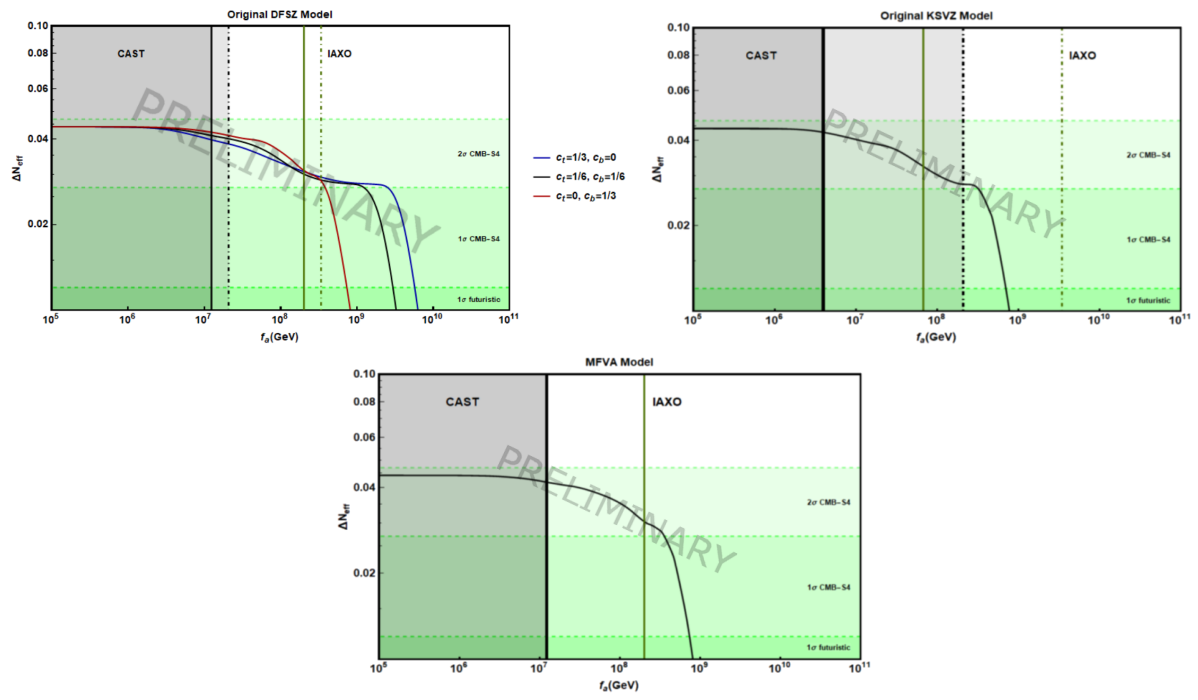

Figure 2: Prediction for $\Delta N_{\text {eff }}$ as a function of $f_{a}$ in the three discussed models. Regions excluded by CAST are shown shaded in grey delimited by black vertical lines, whereas the highest $f_{a}$ IAXO could probe is shown with green vertical lines. Solid vertical lines correspond to the smallest axion-photon coupling in the DFSZ and KSVZ models, where different values are possible, while dot-dashed lines correspond to the largest possible axion-photon coupling.

lower temperatures as it does not involve QCD and can yield a boosted signal, up to $\Delta N_{\text {eff }} \sim 0.3$, which is within the $2 \sigma$ sensitivity region of the latest CMB experiments. In particular, although CMB and LSS data alone do not hint at a non-zero value of $\Delta N_{\text {eff }}$ [24], when including $S H_{0} E S$ 2019 local Hubble constant measurement of $H_{0}$ [25] there is a shift of the central value towards $\Delta N_{\text {eff }}=0.26_{-0.15}^{+0.16}[26]$ (or $\Delta N_{\text {eff }}=0.28_{-0.17}^{+0.16}$ [27] adding also the Pantheon Supernova dataset) which is in remarkable agreement with the above prediction. Such values will be tested also by forthcoming CMB experiments, such as LiteBIRD [16], Simons Observatory [17] and CMB-S4 [15]. Lastly, we will also study the possibility of a DFSZ axion compatible with the XENON1T excess being probed via $\Delta N_{\text {eff }}$, having chosen the charged leptons to couple like down-type quarks.

\section{Acknowledgements}

The author acknowledges partial financial support by the Spanish MINECO through the Centro de excelencia Severo Ochoa Program under grant SEV-2016-0597, by the Spanish "Agencia Estatal de Investigacíon"(AEI) and the EU "Fondo Europeo de Desarrollo Regional" (FEDER) through the projects FPA2016-78645-P and PID2019-108892RB-I00/AEI/10.13039/501100011033.

\section{References}

[1] R. D. Peccei and H. R. Quinn, Phys. Rev. Lett. 38 (1977), 1440-1443

[2] F. Wilczek, Phys. Rev. Lett. 40 (1978), 279-282

[3] S. Weinberg, Phys. Rev. Lett. 40 (1978), 223-226

[4] R. J. Crewther, P. Di Vecchia, G. Veneziano and E. Witten, Phys. Lett. B 88 (1979), 123 [erratum: Phys. Lett. B 91 (1980), 487]

[5] C. A. Baker, D. D. Doyle, P. Geltenbort, K. Green, M. G. D. van der Grinten, P. G. Harris, P. Iaydjiev, S. N. Ivanov, D. J. R. May and J. M. Pendlebury et al., Phys. Rev. Lett. 97 (2006), 131801 [arXiv:hep-ex/0602020] 

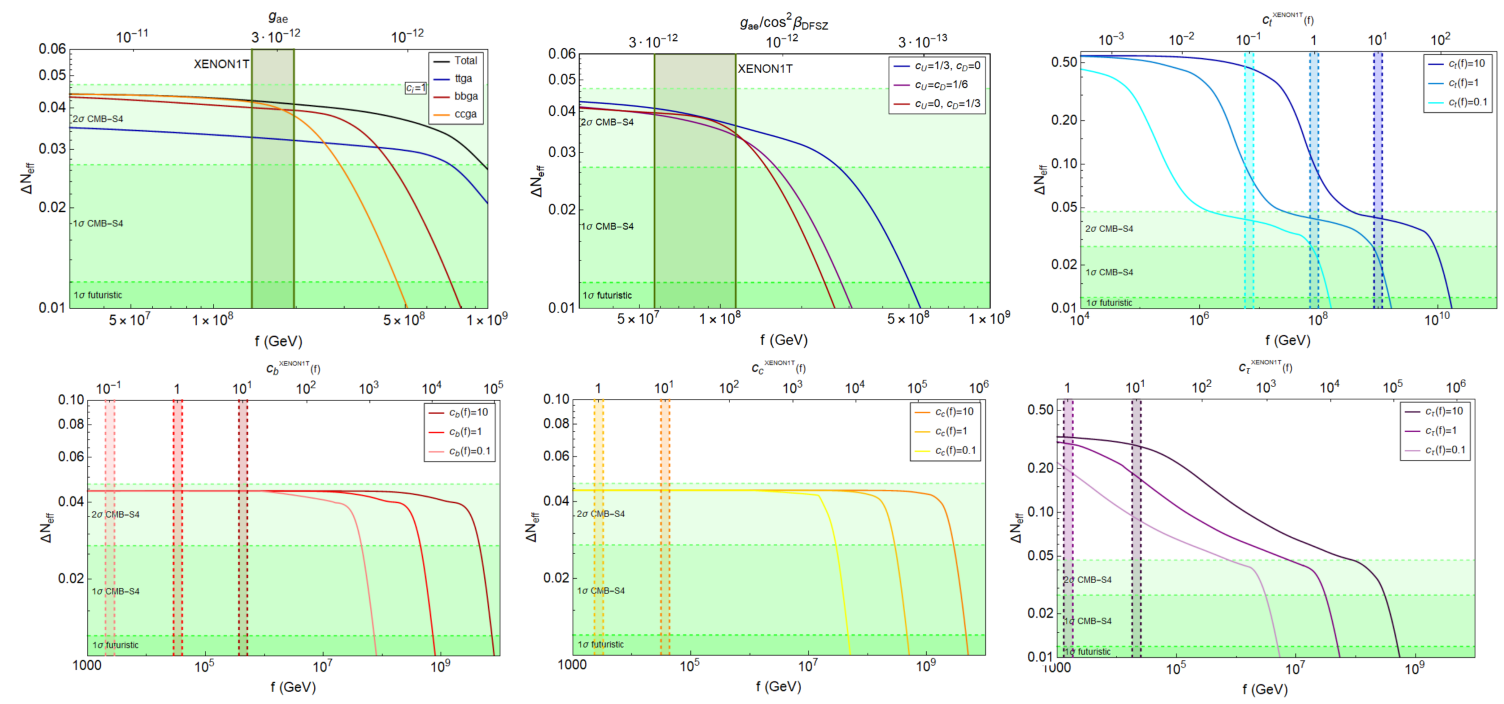

Figure 3: Prediction for $\Delta N_{\mathrm{eff}}$ as a function of $f_{a}$ for axions relevant for the XENON1T excess. In the first row on the left we show the result for the democratic case, with the one for the DFSZ model on the center. The last plot in the first row and those on the second one show the results for the loop-induced electron coupling case. In all cases, the axion initial abundance has been taken as zero and the axion scale windows preferred by the XENON1T collaboration are shown accordingly in each case as vertical shaded bands.

[6] J. M. Pendlebury, S. Afach, N. J. Ayres, C. A. Baker, G. Ban, G. Bison, K. Bodek, M. Burghoff, P. Geltenbort and K. Green et al., Phys. Rev. D 92 (2015) no.9, 092003 [arXiv:1509.04411]

[7] D. J. E. Marsh, Phys. Rept. 643 (2016), 1-79 [arXiv:1510.07633]

[8] A. Arvanitaki, S. Dimopoulos, S. Dubovsky, N. Kaloper and J. March-Russell, Phys. Rev. D 81 (2010), 123530 [arXiv:0905.4720]

[9] XENON Collaboration, E. Aprile et al., Phys. Rev. D 102 (2020) no.7, 072004 [arXiv:2006.09721]

[10] CAST Collaboration, E. Arik et al., JCAP 02 (2009), 008 [arXiv:0810.4482 [hep-ex]]

[11] I. G. Irastorza, F. T. Avignone, S. Caspi, J. M. Carmona, T. Dafni, M. Davenport, A. Dudarev, G. Fanourakis, E. Ferrer-Ribas and J. Galan et al., JCAP 06 (2011), 013 [arXiv:1103.5334 [hep-ex]]

[12] E. Armengaud, F. T. Avignone, M. Betz, P. Brax, P. Brun, G. Cantatore, J. M. Carmona, G. P. Carosi, F. Caspers and S. Caspi et al., JINST 9 (2014), T05002 [arXiv:1401.3233 [physics.ins-det]]

[13] ADMX Collabotarion, S. J. Asztalos et al., Phys. Rev. D 64 (2001), 092003

[14] ADMX Collabotarion, S. J. Asztalos et al., Phys. Rev. D 69 (2004), 011101 [arXiv:astro-ph/0310042 [astro-ph]]

[15] CMB-S4 Collaboration, K. N. Abazajian et al., [arXiv:1610.02743 [astro-ph.CO]]

[16] https://astro.unibonn.de/ kbasu/ObsCosmo/Slides2019/sciencelitebird final.pdf

[17] Simons Observatory Collaboration, P. Ade et al., JCAP 02 (2019), 056 [arXiv:1808.07445 [astro-ph.CO]]

[18] M. Dine, W. Fischler and M. Srednicki, Phys. Lett. B 104 (1981), 199-202

[19] A. R. Zhitnitsky, Sov. J. Nucl. Phys. 31 (1980), 260

[20] J. E. Kim, Phys. Rev. Lett. 43 (1979), 103

[21] M. A. Shifman, A. I. Vainshtein and V. I. Zakharov, Nucl. Phys. B 166 (1980), 493-506

[22] F. Arias-Aragon and L. Merlo, JHEP 10 (2017), 168 [arXiv:1709.07039 [hep-ph]]

[23] F. Arias-Aragon, F. D’Eramo, R. Z. Ferreira, L. Merlo and A. Notari, [arXiv:2007.06579 [hep-ph]]

[24] Planck Collaboration, N. Aghanim et al., Astron. Astrophys. 641 (2020), A6 [arXiv:1807.06209 [astro-ph.CO]]

[25] A. G. Riess, S. Casertano, W. Yuan, L. M. Macri and D. Scolnic, Astrophys. J. 876 (2019) no.1, 85 [arXiv:1903.07603 [astro-ph.CO]]

[26] G. Ballesteros, A. Notari and F. Rompineve, [arXiv:2004.05049 [astro-ph.CO]]

[27] M. Gonzalez, M. P. Hertzberg and F. Rompineve, JCAP 10 (2020), 028 [arXiv:2006.13959 [astro-ph.CO]] 